Boise State University

ScholarWorks

Counselor Education Faculty Publications and

Presentations

Department of Counselor Education

$10-1-2017$

Counselor Allegiance and Client Expectancy in Neuroscience-Informed Cognitive-Behavior Therapy: A 12-Month Qualitative Follow-Up

Thomas A. Field

City University of Seattle

Eric T. Beeson

Northwestern University

Laura K. Jones

University of North Carolina at Asheville

Raissa Miller

Boise State University 


\section{Counselor Allegiance and Client Expectancy in Neuroscience-Informed Cognitive-Behavior Therapy: A I2-Month Qualitative Follow-Up}

Thomas A. Field, Eric T. Beeson, Laura K. Jones, and Raissa Miller

This article presents summative findings from a 12-month multiphase mixed-methods pilot study examining counselor and client perceptions of neuroscience-informed cognitive-behavior therapy $(n C B T)$ following clinical application. Results from the first 6 months of the study indicated that the counselor's and client's beliefs about the credibility and effectiveness of nCBT (i.e., expectancy) remained stable from pretreatment to 6 months into treatment. The fourth phase of data collection at the 12-month interval followed an explanatory sequential process whereby the qualitative data were connected to earlier merged quantitative data to better understand initial findings from the first 6 months of the study. Results indicate that counselors' initial comprehension and familiarity with the model, counselor-client trust, counselor delivery and suggestion, and client willingness to practice outside of session were key components to the development of counselor and client belief (expectancy) in the model. Implications for nCBT theory development and application are discussed.

Practitioners, educators, and supervisors within the counseling field are working to determine effective ways of integrating neuroscience into clinical practice. Currently, there is a dearth of research examining neuroscienceinformed counseling frameworks that can guide working with clients (Beeson \& Field, 2017). Most neuroscience-informed models, such as interpersonal neurobiology, have emerged from outside the counseling field. One of the few counseling theories to incorporate emerging neuroscience research is neuroscience-informed cognitive-behavior therapy (nCBT). The model was first described in the Journal of Mental Health Counseling (Field, Beeson, \& Jones, 2015).

Thomas A. Field, Division of Arts and Sciences, City University of Seattle; Eric T. Beeson, Department of Counseling@Northwestern, The Family Institute at Northwestern University; Center for Applied Psychological and Family Studies, Northwestern University; Laura K. Jones, Department of Health and Wellness, University of North Carolina at Asheville; Raissa Miller, Department of Counselor Education, Boise State University.

Correspondence concerning this article should be addressed to Thomas A. Field, School of Arts and Sciences, City University of Seattle, 521 Wall Street, Seattle, WA 98121. E-mail: tfield@cityu.edu 
The nCBT approach introduces an expanded approach to Ellis's (1962) $\mathrm{ABCDE}$ model, which proposed that as a response to antecedents in a person's environment (A), a person develops beliefs (B) that result in emotional and behavioral consequences $(\mathrm{C})$. The person is then trained to dispute (D) these beliefs, resulting in new emotional and behavioral consequences $(\mathrm{E})$. By integrating knowledge of how the brain processes information differently based upon certain past events and present environmental factors such as threat or pronounced reward, nCBT aims to provide a more robust and broadly applicable approach to clinical practice. Specifically, nCBT uses the two-system process models of Kahneman (2011) and later McRae, Misra, Prasad, Pereira, and Gross (2012) and LeDoux and Pine (2016) for top-down and bottom-up processing, in an effort to build a more representative model for conceptualizing how clients may be responding to activating events and how counselors can best respond to such differential processing. The two stages of the nCBT model, representing bottom-up and top-down processing, are denoted Wavel and Wave2. During the first nCBT A-B-C process, Wavel, the person responds to information that is collected by the senses from the environment and internal sources such as physiology $(\mathrm{Al})$, processes information subcortically and preconsciously $(\mathrm{Bl})$, and experiences resulting emotional and physiological consequences $(\mathrm{Cl})$. Activating events can either be episodic or perpetually occurring phenomena such as experiences of racism and oppression. During the second nCBT A-B-C process, known as Wave2, the person becomes aware of these consequences (A2) and appraises their responding (B2), resulting in secondary emotional and physiological consequences (C2). This process can continue as new stimuli are received from the environment and processed.

The initial empirical testing of the model included a 12-month naturalistic pilot study that sought both to understand how counselors were using the model in clinical practice and to assess counselor and client belief in the model following clinical application (Field, Beeson, \& Jones, 2016). The researchers' decision to examine counselor and client perceptions was driven by the consistent meta-analytic research (e.g., Wampold \& Imel, 2015; Wampold et al., 1997) indicating that a counselor's and client's shared belief in a therapeutic approach influenced the outcome of the intervention more than the specific therapy model selected (Anderson, Lunnen, \& Ogles, 2010). Thus, understanding the development of counselor and client beliefs about the model was a crucial first step to evaluating the effectiveness (i.e., outcome) of the model in future experimental studies. An article that presented data from the first 6 months of the year-long study was published in the Journal of Mental Health Counseling (Field et al., 2016). This article included an analysis of counselor and client belief in the credibility and effectiveness of nCBT, known as expectancy. The present article presents data from a follow-up survey that was administered at the conclusion of the study (i.e., 12-month interval), to better understand the findings from the first 6 months of the study and understand the process by which counselor and client expectancy was developed and sustained during nCBT treatment. 


\section{METHODOLOGY}

A complete description of the research design, participants, instruments, procedures, and data analysis for the 12-month pilot study can be found in Field et al. (2016). The authors also identified important limitations of the study, such as small sample size, naturalistic design, and counselor recidivism ( $n=5$; counselor sample size was $n=19$ at the 3 -month interval). To summarize, the researchers recruited counselors for the study, through convenience sampling, who (1) had experience providing cognitive-behavioral therapy (CBT) and (2) were currently working in clinical practice (i.e., seeing clients). Participants were recruited at the conclusion of one of three $\mathrm{nCBT}$ workshops, one at a national counseling convention and two in a large city in the U.S. Northwest. The initial sample of practitioner participants ( $n=$ 24) primarily featured counselors $(n=23,95.8 \%)$ who were overwhelmingly located in the U.S. Northwest $(n=23,95.8 \%)$; mostly held master's degrees $(n=23,95.8 \%)$; were predominantly female $(n=21,87.5 \%)$; worked primarily in private practice $(n=17,70.8 \%)$ and community mental health centers $(n=4,16.7 \%)$; ranged in age from 30 to 64 years $(M=45.74, S D=10.86)$; and were Euro-American (58.3\%, $n=14$ ), Asian and Asian-American (16.7\%, $n=4)$, Latino/a $(8.3 \%, n=2)$, African and African-American $(4.2 \%, n=1)$, and "other ethnicity" $(8.3 \%, n=2)$. As might be expected, considering that prior experience with CBT was an inclusion criterion for the study, $75 \%$ of participants $(n=18)$ identified CBT as one of their theoretical approaches. To reduce allegiance bias, all counselors were either fully licensed or licensure candidates who were supervised by a person unrelated to the study. No direct supervision was provided to participants, though two group consultation meetings were provided to support implementation.

The researchers used a multiphase mixed-methods design (Creswell \& Plano-Clark, 2011), merging qualitative with quantitative data at four intervals $(0,3,6$, and 12 months posttraining). The researchers selected a mixedmethods design because quantitative and qualitative data were intentionally collected concurrently throughout the study, and the collection of either form of data alone would have limited study findings. During the first 6 months of the study, quantitative data were collected through the Credibility/Expectancy Questionnaire (CEQ; Borkovec \& Nau, 1972; Devilly \& Borkovec, 2000) to measure the counselor and client's perceived belief in the credibility of the theoretical model (rationale credibility), as well as expectations for client improvement (expectancy) following implementation. Both counselors $(n=$ 24 ) and clients $(n=35)$ completed this instrument before treatment, 3 months into treatment, and 6 months into treatment. During the first 6 months of study, qualitative data were also collected from open-ended questions on a survey that accompanied the CEQ instrument. Counselor participants also collected client CEQ ratings during the first session of treatment, at 3 months into treatment, and at 6 months into treatment. At the 3 - and 6-month intervals, counselors sent these client ratings to the primary researcher, alongside the counselors' responses to the qualitative survey and CEQ instrument. 
Qualitative data from the counselor survey administered at 0,3 , and 6 months were analyzed via consensus coding and categorization following the constant comparative method from grounded theory research (Glaser \& Strauss, 1967), identifying themes in the data and continuing until the data approached saturation or redundancy (Lincoln \& Guba, 1985). Categorized qualitative data were then transformed into quantitative data via frequency counts and merged with quantitative data from the counselor CEQ, to better understand the meaning of these ratings. The researchers conducted an analysis of variance statistical procedure for counselor CEQ ratings at 0-, 3-, and 6-month intervals, and for client ratings at 0 - and 3-month intervals, to ascertain whether significant differences existed across intervals. Correlational statistics were also performed for counselor and client CEQ ratings at 0 and 3 months. Quantitative data were given higher weighted priority, as qualitative data were used to better understand the quantitative data.

Initial quantitative results indicated that counselor and client ratings were consistently high and did not vary significantly between intervals, and yet counselor and client CEQ ratings were more closely correlated at 3 months $(r=.63)$ than at 0 months $(r=.19)$. In response to these findings, the researchers modified the fourth and present phase of the multiphase design by developing and reviewing follow-up questions.

The fourth and present phase of data collection (12-month interval) followed an explanatory sequential process whereby the qualitative data were connected to earlier merged quantitative data to better understand initial quantitative findings. Explanatory sequential designs are common in mixed-methods research and can be incorporated into multiphase designs (Creswell \& PlanoClark, 2011). Because of the longitudinal nature of multiphase mixed-methods studies, it is not uncommon for the researcher to modify the later data collection processes in response to earlier findings (Creswell \& Plano-Clark, 2011).

To initiate the present phase of research, the first author sent counselors and clients a follow-up survey of four qualitative open-ended questions to better understand findings from the first 6 months of the study. Follow-up questions also sought to understand the process by which counselors and clients developed and sustained a belief in the credibility and effectiveness of nCBT. Faculty and students at the first author's university piloted the finalized questions.

A similar procedure to that employed in the first 6 months of the study was used to collect data for the 12-month qualitative follow-up. Counselors mailed completed responses to the qualitative survey, both for themselves and for clients. Responses were aggregated into a spreadsheet for coding, and a coding consensus team comprising the authors analyzed the data. The coding consensus team included both emic and etic perspectives. The first and second authors (Field and Beeson) had emic positioning, as they conducted the trainings from which participants were recruited. The first author also retained contact with counselor participants throughout the study. The third and fourth authors (Jones and Miller) had etic positioning, with no exposure to participants during the study. This balance reduced the likelihood that bias would 
be interjected into the analysis of qualitative data. Coding proceeded using the same codebook established during the first 6 months of the study. Codes were categorized, and all coding team members reviewed the resulting expectancy process model, described later in the manuscript, to ensure consistency with the data. The first author kept an audit trail throughout the 12-month study to enhance the trustworthiness of the data.

\section{RESULTS}

Most of the remaining counselors in the study completed the follow-up questionnaire ( $n=17,89.5 \%$ of the 19 counselors from the 6 -month interval), with 10 of their clients completing the survey (a $28.6 \%$ response rate from the 35 clients who received nCBT during the study).

From analysis of qualitative data, a pathway was identified by which counselors and clients developed beliefs that change would occur during nCBT treatment (i.e., expectancy). Figure 1 depicts the process of nCBT expectancy development. The model outlines the development of counselor belief in the credibility of the model (allegiance), as well as how the counselor's communication of nCBT's relevance to the client within the context of a trusting relationship influences the client's willingness to participate in nCBT. From the qualitative follow-up survey administered to counselors and clients, several stages are identified in this expectancy process model: (1) counselor understanding and allegiance; (2) counselor-client trust and counselor delivery, suggestion, and salesmanship; (3) client understanding, willingness, and expectancy; and (4) results and outcomes. We describe each stage of the expectancy process.

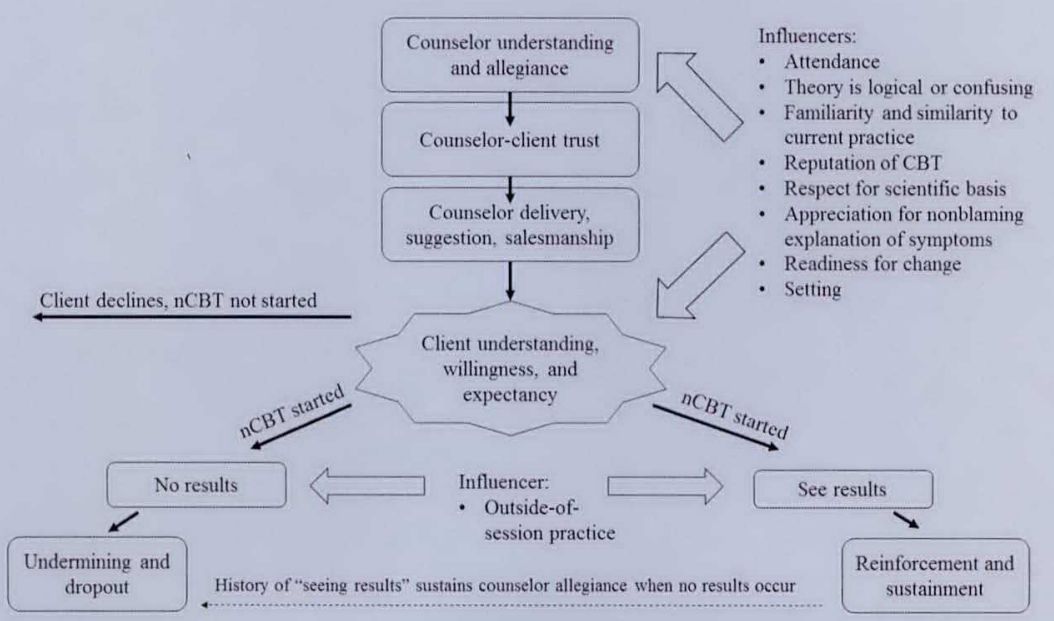

Figure I. The neuroscience-informed cognitive-behavior therapy (nCBT) expectancy process model for counselors and clients. CBT = cognitive-behavioral therapy. 


\section{Counselor Understanding and Allegiance}

Counselors reported that the client's belief in the credibility and effectiveness of nCBT was strongly related to the counselor's own allegiance to the method. The counselor's belief was initially developed at the point of training. Several factors seemed to impact counselor allegiance, including attending an nCBT training, perceiving the theory as logical or confusing, having familiarity and similarity with current practices, believing in the reputation of CBT, respecting the scientific basis of neuroscience, appreciating the nonblaming explanation of symptoms, being ready to adopt a new model of therapy, and practicing in a setting conducive to nCBT.

Counselors also believed that attending a training and understanding the method were foundational to the development of their own allegiance to the method. When the counselor experienced the theory as "logical" during training, they felt prepared to explain and deliver the method with clients because they first understood it themselves. Being able to describe nCBT in a coherent way to clients seemed very important to client buy-in. When counselors were confused by the concepts, their lack of comprehension resulted in being confusing when trying to introduce the concepts to clients. As a result, both the counselor and their clients then had little initial belief that nCBT would be effective. When counselor and client felt confused, both gave up quickly on the modality.

Preexisting theoretical orientations shaped counselor beliefs about the model's effectiveness. The majority of counselors $(75 \%, n=18)$ identified CBT as their theoretical orientation. Other counselors possessed a theoretical orientation that was close to CBT in terms of its structured, goal-directed nature. For example, one counselor stated, "My theoretical orientation is closer to brief therapy than psychoanalytic. I view therapy as like training wheels-the goal is helping clients learn how to manage problems on their own."

CBT's reputation as being empirically supported for symptom relief seemed to lend credibility to the new neuroscience-informed method. Other counselors appreciated neuroscience and were more open to the modality because of its integration of neuroscience.

Other counselors did not have an overt CBT theoretical orientation, though their approach was still commensurate with nCBT. Some counselors reported that they were already explaining to clients how physiology (bodily response) often impacts thoughts, behaviors, and emotions. One counselor developed immediate allegiance to nCBT because they felt it was the "confirmation of techniques I had already been using." Many of these counselors identified their clinical approach as somewhat eclectic, incorporating elements of yoga, bodywork, and non-Western approaches such as Morita therapy. One of these counselors believed that nCBT "opens the door to person-specific treatment" and understood that each person needs a slightly different treatment approach. In this regard, nCBT was favorably perceived as more flexible and broadly applicable than traditional CBT.

Counselors whose approach was substantially different from CBT often experienced dissonance with applying $\mathrm{nCBT}$ with clients and wondered if 
this dissonance impacted client buy-in and commitment to the method. One counselor self-identified as aligning more with attachment and transpersonal approaches than CBT, and as a result, felt that nCBT was "a vast leap forward" compared to CBT, though "there are still missing pieces that contribute to healing, so it feels lacking." This counselor's clients were often unwilling to complete homework assignments outside of session, and the counselor wondered if her dissonance with the CBT orientation may have influenced her description and delivery (i.e., psychoeducation) of the outside-of-session homework assignments, which she imagined would have impacted client commitment to complete those assignments.

Counselors tended to value the $\mathrm{nCBT}$ approach to psychoeducation, as they found that it further developed rapport by reducing clients' shame and self-blame for their symptoms. The nCBT model was perceived to validate client struggles with trying to control automatic processes, rather than attributing responsibility to the client for dysregulation and believing that they "should" be able to control their responding at all times. Counselors understood that in many cases, cognitive control of emotions and physiology is not possible, as the brain and body have learned adaptive mechanisms for responding automatically.

Other notable influencers on the development of counselor allegiance to nCBT included the counselor's readiness to adopt a new model of therapy and whether their practice setting was conducive to nCBT. For example, one of the counselors in the study was working in an emergency department and found $\mathrm{nCBT}$ to be useful for brief, episodic encounters with clients struggling with acute symptoms. However, this counselor also believed that nCBT would likely be more effective in outpatient settings that afforded both longer session time and greater frequency of sessions for the counselor to monitor the client's outside-of-session practice.

\section{Counselor-Client Trust and Counselor Delivery, Suggestion, and Salesmanship}

Both counselors and clients felt that counselor-client trust had to be established in order for the counselor's allegiance to influence the client's expectancy. When a strong therapeutic relationship was established, the client was more willing to trust the counselor's suggestion that nCBT could be helpful for their current problems. This "salesmanship" provided both an explanation for client symptoms and a rationale for addressing those symptoms. Counselors emphasized that time was required to establish the therapeutic relationship and provide the psychoeducation needed.

Several clients in the study confirmed that their own willingness to participate in nCBT treatment was strongly influenced by their trusting relationship with a counselor who believed in nCBT and communicated this to them. One client shared that "the ideas seemed weird to me ... I guess I trusted my doctor and did it anyway. She said these tricks might work." This initial skepticism was shared by another client, who also decided to participate in nCBT because 
of their trust in their counselor's suggestion: "When I was first given tools to combat triggers, I was skeptical. They seemed unrelated to my problems. My therapist seemed to believe in her ideas, so I took [them] on faith and went ahead with the program." Counselors believed that skepticism could be overcome through a trusting relationship: "I think when a trusting relationship is there, the client is more apt to engage and 'buy into' what is being presented and utilized by the clinician."

In the beginning, the counselor's confidence, belief (allegiance), and delivery all impacted client expectancy enough for the client to try the method. When the client agreed to try the method, the alliance of the shared objective between the counselor and client helped to further establish both parties' belief in the method. When the client later saw positive results from the intervention, it validated their trust in the counselor.

\section{Client Understanding, Willingness, and Expectancy}

The client's trust in the counselor's suggestion of nCBT appeared to be the most crucial factor in client willingness to participate in nCBT. Once a counselor understood the modality and developed an allegiance to it, their delivery of the rationale for $\mathrm{nCBT}$ and their communication of expected change occurring to the client were crucial to client buy-in. Clients who trusted their counselor were willing to try the modality and override their skepticism. The client's willingness to participate in nCBT and the development of their expectancy were also influenced by similar factors to those that had influenced the counselor earlier: attending sessions, perceiving the theory as logical or confusing, having familiarity and similarity with current practices, believing in the reputation of CBT, respecting the scientific basis of neuroscience, appreciating the nonblaming explanation of symptoms, being ready to change, and practicing in a setting conducive to nCBT. For example, one counselor reported that the scientific basis of the psychoeducational material helped clients respond favorably: "My few clients that I used this approach with really seemed to simply calm down, after learning the science behind the thought-behavior reaction."

As with counselors, clients' initial response to $\mathrm{nCBT}$ was strongly influenced by prior experiences and familiarity with similar methods. Clients who had responded positively to practices like mindfulness or yoga also responded positively to nCBT when it was first introduced. For example, one client learned similar ways of coping through yoga practice, and thus "was an easy sell ...I believed in the study right away." Assessing the client's preexisting familiarity with similar methods therefore appears important when introducing nCBT.

Some clients were skeptical of biofeedback and neurofeedback interventions. One counselor preferred interventions that did not require the client to purchase equipment for practicing at home, reporting that clients were "leery" of technologically based interventions such as at-home biofeedback devices. Their clients were suspicious that the counselor was receiving "kickbacks" from the biofeedback equipment company to promote their product. This 
counselor believed that client skepticism in purchasing devices for home use derived from the ongoing outcry concerning the influence of the pharmaceutical industry in physician prescribing practices. Some clients apparently have become skeptical even about receiving counselor recommendations for medication evaluation, believing pharmaceutical companies to have a pernicious influence on referral practices. Despite this skepticism, clients were willing to try these interventions because of their trust in the counselor. Counselors who want their clients to practice technologically based interventions outside of session should address any preexisting concerns about counselor conflict of interest, as counselor suggestion of these methods could engender greater client skepticism than the suggestion of methods that do not require the client to purchase equipment for home use.

\section{Results and Outcomes}

As the clients became more familiar with the approach and started implementing the techniques in their life through repeated practice, many "saw results." One client reported that "after 3 months, people could notice a change in my condition." Improvements included gaining a repertoire of coping skills for when clients felt overwhelmed, using new skills "without hesitation," noticing triggers "on my own," generalizing these gains to their personal life without the therapist's constant directives, and thus becoming "more confident in going it alone." One client particularly benefited from the Wavel nCBT intervention of tracking her pulse. The counselor reported that this technique helped her remain grounded rather than ruminative. As a result, her counselor reported that "she wasn't lost in the ether." The majority of clients shared that being able to relax through attending to stimuli in their current environment rather than engaging in unhelpful response styles when triggered (e.g., avoidance) was the most helpful component of nCBT, and they were pleased to find that this skill was useful even in crisis situations.

Positive outcomes seemed crucial to the counselor's sustained expectancy. Clients were empowered by these gains, and counselors believed they were facilitating their client's empowerment. Thus, experiencing $\mathrm{nCBT}$ as clinically effective appears to sustain and reinforce the counselor and client's belief in nCBT"s credibility and effectiveness. Positive client outcomes also bolstered the strength of the therapeutic relationship by validating the counselor's earlier salesmanship that the intervention could be helpful to the client.

Positive outcomes appeared to protect counselor allegiance and expectancy even when client-neutral outcomes occurred. Counselors appeared to understand that no single approach worked for all clients, and they balanced client-neutral outcomes in light of client-positive outcomes. One counselor in the study indicated that their belief in nCBT would have been negatively impacted if their clients experienced "ill effects" (negative outcomes) from the intervention. No counselors reported negative outcomes of nCBT.

Both counselors and clients expressed the belief that "hard-wiring takes time to undo," and that repetitive practice was essential to learning new 
responses to triggers such as perceived threats or drug cravings. Counselors considered nCBT to be an approach that engaged clients "in taking an active role in treatment" through tasks such as practicing skills outside of sessions. The requirement for outside-of-session practice received a mixed response from clients. For example, some clients found it helpful to complete the worksheet for logging physiological responses and associated thoughts, feelings, and behaviors. One client diagnosed with an autism spectrum disorder found the concrete nature of logging physiological response to be helpful in his development of physiological and interoceptive awareness. Another client had dependency issues that were aggravated by a recent divorce summons. She had become dependent on the counselor for advice and guidance when dysregulated, and the worksheet gave her a task to complete rather than constantly contacting the counselor. Through completing the worksheet, the client learned to sit with their emotions before acting on them and became more autonomously self-regulated.

However, not all clients participated in completing therapeutic tasks outside of sessions. Counselors reported that some clients were not ready to change, and others were resistant to completing outside-of-session practice. Clients resisted actively engaging in treatment tasks for a variety of reasons, such as lacking an understanding of the treatment approach, lacking the expectancy that it could be helpful, not having the time outside of sessions to complete homework assignments, avoiding acknowledging personal responsibility, fearing detrimental effects, and fearing failure as a result of trying something new. Some counselors reported that very few clients actually completed outside-of-session practice. Although counselors and clients understood repetitive practice to be helpful in the development of new skills, requiring clients to practice every day was perceived to be potentially detrimental to the overall success of the intervention, as clients would have been set up for failure:

If the person is meant to practice the tool every single day, and doesn't practice one day, they may feel like a failure. They may quit or start to think that something is wrong with them. The nCBT approach is flexible, not fear-based, and doesn't encourage fatalistic thinking.

The counselor's delivery of the treatment method was crucial to the client's willingness to practice outside of session. When the client trusts the counselor and observes the counselor's allegiance to an intervention, they are far more likely to at least try the suggested intervention. As one client reported, "My therapist was confident her strategies could work. Her belief helped me decide to try the strategies for myself."

\section{DISCUSSION}

From analyses of the qualitative data, a process of counselor and client expectancy was identified whereby (1) the counselor clearly understands the model and develops allegiance to it; (2) the counselor communicates their belief that the model will be helpful to the client, within the context of a trust- 
ing therapeutic relationship; (3) the client has faith and trust in the counselor and is willing to participate in nCBT despite possible skepticism; (4) the client sees results, which validates and reinforces their trust in the counselor; and (5) the counselor's overall allegiance is reinforced and becomes resilient even in the face of neutral client outcomes. These five steps appear to be the proximal pathway to nCBT expectancy. Several factors can interrupt this process, such as when the counselor does not understand the model and thus cannot explain it effectively to clients, or when insufficient trust exists between counselor and client. The counselor and client's expectancy is also influenced by other distal factors, such as attending trainings and sessions, perceiving the theory as logical or confusing, having familiarity and similarity with current practices, believing in the reputation of CBT, respecting the scientific basis of neuroscience, appreciating the nonblaming explanation of symptoms, being ready to change, and practicing in a setting conducive to nCBT.

\section{Implications for Training}

Counselor understanding, allegiance, and suggestion seem to strongly influence the development of client expectancy. It thus seems important that counselors understand the nCBT model and believe it to be effective. Trainings should include an assessment of practitioner understanding of the model and provide any needed follow-up through consultation or supervision. When counselors do not understand the nCBT model, their belief in its effectiveness diminishes, and they struggle to communicate the model effectively to clients when providing psychoeducation. Clients then struggle to understand the model themselves, reducing their willingness to participate in $\mathrm{nCBT}$ and their expectancy that nCBT will help them. One counselor lacked an understanding of the model and subsequently struggled to communicate the model to their clients, confusing them. As a result, both the counselor and their clients felt confused and gave up quickly on the modality.

It is noteworthy that the two other counselors in the study who dropped out early (i.e., before the 3-month interval) were the only two participants who scored less than $80 \%$ on the five-item posttraining nCBT knowledge assessment. The initial training therefore appears vitally important to the development and sustainment of counselor allegiance and expectancy, as early confusion can result in counselors failing to develop sufficient allegiance to continue using the model in practice. Compared to other influencers on counselor allegiance, training also appears to be more easily modifiable. Many of the factors that influenced the development of counselor allegiance appear to be intrinsically motivated and difficult to change, such as familiarity and similarity with current practices, believing in the reputation of CBT, respecting the scientific basis of neuroscience, appreciating the nonblaming explanation of symptoms, being ready to adopt a new model of therapy, and practicing in a setting conducive to nCBT. This finding further emphasizes the importance of ensuring that the training is of sufficient quality and duration for counselors to develop an allegiance to $\mathrm{nCBT}$. 
Once a counselor understands nCBT, their development of allegiance to the method is crucial to the effectiveness of later actions such as communicating a belief to the client that nCBT can be helpful for their presenting symptoms. Because of this importance, trainings should include self-assessments of the counselor's own belief in the method following training. Counselors could practice asking themselves if they believed $\mathrm{nCBT}$ would be helpful with particular client presentations. This information could inform the counselor's rationale for why nCBT would be helpful in addressing the client's problems. Our findings are consistent with existing literature on the importance of counselors understanding the treatment approach as a step toward developing allegiance in the method (Wampold \& Imel, 2015).

Training length. Whereas counselors had mixed opinions about optimal training length at both the initial posttraining assessment and the 12-month follow-up, it seems likely that nCBT training needs to be longer and more in-depth than the 3-hour training used for this preliminary study. A 2-or 3-day training (i.e., 12-18 hours) may help to ensure that all counselors understand the model and feel prepared to use it in practice settings. During nCBT training, counselors should understand that forging a trusting therapeutic bond with the client is the first step in the treatment process. Introducing the nCBT model as a potential treatment approach should proceed only after rapport has been established, as clients may respond with some degree of skepticism to this suggestion. When clients trust their counselors, it appears to overrule their skepticism, and they become willing to try the modality. However, although introducing $\mathrm{nCBT}$ before trust has been established has the potential to be ineffective, the exact timing remains unclear and could be examined in further studies. It is likely that the timing of the counselor's suggestion depends upon the counselor's ability to establish rapport quickly, alongside the client's openness and propensity toward trusting professionals. Because the exact timing of the counselor's suggestion of using nCBT may depend on several factors, counselors should individualize their approach to each client, informed by the counselor's analysis of the quality of the relationship. Trainings should emphasize the importance of developing a trusting relationship and respecting the trust that clients have in their counselors.

Counselor suggestion. Counselors' communication of their belief in the credibility and effectiveness of $\mathrm{nCBT}$ seemed crucial to client buy-in, and future trainings could include guidance for how to provide a rationale regarding the relevance of nCBT for specific client complaints. Future trainings could also include guidance on how the counselor can deliver psychoeducation in a manner that is likely to result in client comprehension and expectancy, such as the use of visuals and metaphors to understand Wavel and Wave2 processes. Clients are more likely to grasp Wavel and Wave2 concepts if the counselor speaks in a clear and understandable manner that distills neuroscience information without diluting it.

Client skepticism was common, and outside-of-session practice appeared to be a significant barrier to client improvement and subsequent positive outcomes. Some clients did not complete required outside-of-session practice. 
Client nonadherence to outside-of-session practice assignments was consistent with prior CBT studies that have found homework adherence rates of approximately 40\% (e.g., Decker et al., 2016). This nonadherence is significant when considering that clients who practiced outside of session saw beneficial effects, consistent with meta-analyses that have found outside-of-session practice to approach a medium-size effect $(d=0.48)$ on CBT outcome when compared with treatments that did not include a homework component (Kazantzis, Whittington, \& Dattilio, 2010). Clients did not complete outside-of-session practice for several reasons. Some of these reasons appeared difficult to influence (e.g., insufficient time or resources for outside-of-session practice), whereas others could be addressed directly by the counselor prior to initiating an outside-of-session protocol. For example, counselors could check in with clients about their understanding of the outside-of-session practice protocol to ensure the client comprehends the directions and procedure. The counselor could also directly inquire about any client fears of detrimental effects or fears of failure when trying something new. These discussion points could also be part of subsequent follow-up conversations with the client about their outside-of-session practice. The counselor's intentional discussion of client comprehension and fears could enhance the likelihood that the client will complete outside-of-session practice assignments. Future trainings should consider addressing how best to prepare clients for outside-of-session practice.

\section{Implications for Theory Development}

Throughout the past 12 months of the study, a theme consistently emerged of counselors wanting more interventions and techniques to use with clients. These counselors felt that the psychoeducational part of the model was stronger than the tools currently available for clients to practice outside of sessions. During subsequent model development, it appears that the intervention protocols for the method can be expanded in both depth and breadth.

\section{Limitations}

Limitations of the pilot study were detailed in the former article by Field et al. (2016), and included the overrepresentation of female private practitioners in the counselor sample, small sample size, use of convenience sampling, and low generalizability of findings due to the lack of randomization and control characteristic of a naturalistic study. Future research is needed regarding the potential varying effectiveness of $\mathrm{nCBT}$ with divergent populations. Counselor and client attrition occurred during the study, including further dropout at the 12-month follow-up period. This may have skewed results, as counselors and clients who responded less favorably to nCBT may have left the study before their responses could be collected and included in the analysis. Of note, one counselor who did not have a favorable response to $\mathrm{nCBT}$ remained in the study until its completion. Although nCBT shows considerable promise, additional research into its effectiveness is necessary to further establish the model within counseling and broader mental health fields. All authors and 
research team members had some allegiance bias to the model, since they are also the model's developers. Future experimental studies and study analyses will eventually need to incorporate and be performed by researchers without a direct affiliation with the model, to further reduce the potential confound of researcher allegiance bias.

\section{CONCLUSION}

The nCBT model appears to be an approach that resonates with counselors and clients, and seems to be effective once the counselor and client develop a belief in its credibility and an expectancy that change will occur when using the method. In this article, we described the development of both counselor and client expectancy, with an analysis of factors that seemed to help and hinder its development. Counselors should understand this expectancy development process during subsequent trainings, as the client's willingness to participate in nCBT treatment and complete outside-of-session practice appears strongly influenced by the counselor's development of allegiance and the counselor's communication of their belief in the method. Future treatment manuals could also include information about the expectancy process. This addition would also ensure that all counselors who provide nCBT in future experimental trials are trained to attend to the development of expectancy, which is believed to enhance willingness to participate in the method and subsequent achievement of positive therapeutic outcomes. 


\section{REFERENCES}

Anderson, T., Lunnen, K. M., \& Ogles, B. M. (2010). Putting models and techniques in context. In B. L. Duncan, S. D. Miller, B. E. Wampold. \& M. A. Hubble (Eds.), The heart and soul of change: Delivering what works in therapy (pp. 143-166). Washington, DC: American Psychological Association.

Beeson, E. T., \& Field, T. A. (2017). Neurocounseling: A new section of the Journal of Mental Health Counseling. Joumal of Mental Health Counseling, 39, 71-83. doi:10.17744/mehc.39.1.06

Borkovec, T. D., \& Nau, S. D. (1972). Credibility of analogue therapy rationales. Journal of Behavior Therapy and Experimental Psychiatry, 3, 257-260. doi:10.1016/0005-7916(72)90045-6

Creswell, J. W., \& Plano-Clark, V. L. (2011). Designing and conducting mixed methods research $\left(2^{\text {nd }}\right.$ ed.). Thousand Oaks, CA: Sage.

Decker, S. E., Kiluk, B. D., Frankforter, T., Babuscio, T., Nich, C., \& Carroll, K. M. (2016). Just showing up is not enough: Homework adherence and outcome in cognitive-behavioral therapy for cocaine dependence. Journal of Consulting and Clinical Psychology, 84, 907-912. doi: $10.1037 / \mathrm{ccp} 000126$

Devilly, G. J., \& Borkovec, T. D. (2000). Psychometric properties of the credibility/expectancy questionnaire. Journal of Behavior Therapy and Experimental Psychiatry, 31, 73-86. doi:10.1016/ S0005-7916(00)00012-4

Ellis, A. (1962). Reason and emotion in psychotherapy. New York, NY: Stuart.

Field, T. A., Beeson, E. T., \& Jones, L. K. (2015). The new ABCs: A counselor's guide to neuroscience-informed cognitive-behavior therapy. Journal of Mental Health Counseling, 37, 206-220. doi:10.17744/mehc.37.3.02

Field, T. A., Beeson, E. T., \& Jones, L. K. (2016). Neuroscience-informed cognitive-behavior therapy in clinical practice: A preliminary study. Journal of Mental Health Counseling, 38, 139 154. doi: $10.17744 /$ mehc. 28.2 .05

Glaser, B. G., \& Strauss, A. (1967). The discovery of grounded theory. Chicago, IL: Aldine.

Kahneman, D. (2011). Thinking fast and slow. New York, NY: Farrar, Straus, \& Giroux.

Kazantzis, N., Whittington, C., \& Dattilio, F. (2010). Meta-analysis of homework effects in cognitive and behavioral therapy: A replication and extension. Clinical Psychology: Science and Practice, 17, 144-156. doi:10.1111/j.1468-2850.2010.01204.x

LeDoux, J. E., \& Pine, D. S. (2016). Using neuroscience to help understand fear and anxiety: A two-system framework. American Journal of Psychiatry, 173, 1083-1093. doi:10.1176/appi. ajp. 2016.16030353

Lincoln, Y. S., \& Guba, E. G. (1985). Naturalistic inquiry. Thousand Oaks, CA: Sage.

McRae, K. M., Misra, S., Prasad, A. K., Pereira, S. C., \& Gross, J. J. (2012). Bottom-up and topdown emotion generation: Implications for emotion regulation. Social Cognitive and Affective Neuroscience, 7, 253-262. doi:10.1093/scan/nsq103

Wampold, B. E., \& Imel, Z. E. (2015). The great psychotherapy debate: The evidence for what makes psychotherapy work (2 ${ }^{\text {nd }}$ ed.). Mahwah, NJ: Lawrence Erlbaum.

Wampold, B. E., Mondin, G. W., Moody, M., Stich, F., Benson, K., \& Ahn, H. (1997). A metaanalysis of outcome studies comparing bona-fide psychotherapies: Empirically, "all must have prizes." Psychological Bulletin, 122, 203-215. doi:10.1037/10033-2909.122.3.203 